POSTERS 


\title{
Reduced MHD and Astrophysical Fluid Dynamics
}

\author{
Wayne Arter \\ EURATOM/CCFE Fusion Association, Culham Science Centre, \\ Abingdon, Oxon. OX14 3DB, UK \\ email: wayne.arter@ccfe.ac.uk
}

\begin{abstract}
Recent work has shown a relationship between between the equations of Reduced Magnetohydrodynamics (RMHD), used to model magnetic fusion laboratory experiments, and incompressible magnetoconvection (IMC), employed in the simulation of astrophysical fluid dynamics (AFD), which means that the two systems are mathematically equivalent in certain geometries. Limitations on the modelling of RMHD, which were found over twenty years ago, are reviewed for an AFD audience, together with hitherto unpublished material on the role of finite-time singularities in the discrete equations used to model fluid dynamical systems. Possible implications for turbulence modelling are mentioned.
\end{abstract}

Keywords. methods: numerical, convection, MHD, turbulence

\section{Introduction}

The author (Arter 2010) has recently shown that there is a close relationship between the equations of high- $\beta$ Reduced Magnetohydrodynamics (RMHD), specified in e.g. Hazeltine \& Meiss $(1992, \S 7)$ and incompressible magnetoconvection (IMC) described in e.g. Proctor (2005). Indeed, the two systems are mathematically equivalent in certain geometries. This paper describes implications of the equivalence for the simulation of AFD applications (of which IMC is one example), from what is known about RMHD, which is used to model magnetic fusion laboratory experiments.

The equivalence between the two systems arises in models with two space dimensions. In RMHD, the presence of a strong magnetic field $\mathbf{B}_{\mathbf{0}}$, leads to an ordering in which fluid flow is incompressible in planes normal to $\mathbf{B}_{\mathbf{0}}$. If further, at leading order, field lines are curved in the manner typical of laboratory magnetic confinement configurations, an effective gravity appears in the model proportional to the local field line curvature. In IMC, both incompressibility and the corresponding buoyancy force term due to gravity arise from the Boussinesq approximation, which is well described in Tritton (1998, $§ 14 \mathrm{~A})$. There are important physical differences, in that in RMHD, the energy source is primarily the background field $\mathbf{B}_{0}$, and the 'gravity' force may be stabilising or destabilising, whereas in IMC, thermal buoyancy is the main driver of instability.

One aspect pioneered in the modelling of laboratory plasmas was the study of the limitations on numerical simulation when dissipation is small, or equivalently at large Reynolds number. Such a regime is dominated by nonlinear effects, so the exact nature of the primary instability is less important.

\section{Nonlinear instability in RMHD}

The paper by Eastwood \& Arter (1986) pointed out that the numerical divergence observed in simulations of RMHD could be explained by nonlinear numerical instability. 


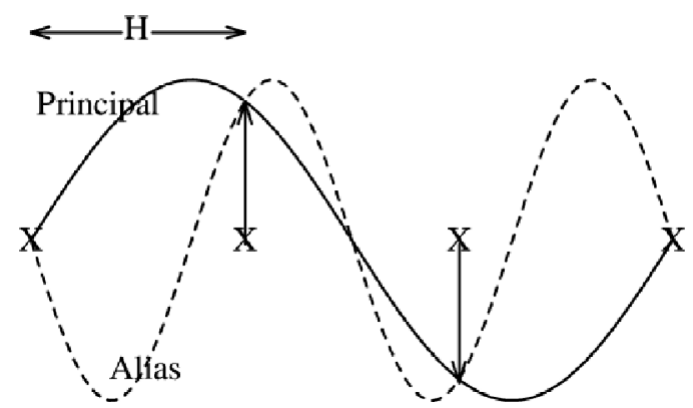

Figure 1. Quadratic nonlinearities cause wavelength $\lambda_{0}=3 H$ to generate a component with $\lambda_{1}=3 H / 2$. When sampled on the mesh (crosses), these both contribute (shown by arrows) to mesh-resolved wavelength $3 H$.

The numerical algorithms used in these codes do not conserve energy and further numerical work by Eastwood \& Arter (1987a) confirmed that field energies actually grew explosively as $\left(t_{0}-t\right)^{-2}$ prior to code termination at time $t=t_{0}$. Increasing the dissipation in the models removed the divergence and led to physically distinct, oscillatory behaviour. A physical explanation for the process is illustrated in Figure 1.

Although this 'alias-feedback' instability is an important issue for old RMHD codes, the above argument is neither rigorous nor quantitative. Since instability occurs even with vanishingly small timesteps, semi-discrete models, continuous in time, for RMHD were studied by Eastwood \& Arter (1986). The 2-D limit of constant pressure was considered, so the results also apply directly to an IMC model. Computer algebra was used to produce semi-discrete models with a spectral discretisation in one co-ordinate and a f.d. (finite difference) representation in the other. The number of degrees of freedom was very limited, typically to three mesh-points and five wavenumbers.

Results were obtained for the resulting RMHD/IMC discretisations which can be understood in terms of the following heuristic model, from Arter \& Eastwood (1987). Suppose $v$ and $b$ represent the velocity and magnetic field respectively, then all the nonlinearities are represented in

$$
\begin{aligned}
& \dot{b}=-b+v b, \\
& \dot{v}=-p_{m} v+v^{2}+b^{2}
\end{aligned}
$$

where the Prandtl number $p_{m}=\nu / \eta$ is the ratio of viscous diffusivity $\nu$ to magnetic diffusivity $\eta$. It will be seen that the origin in $(b, v)$ space is attracting for sufficiently small initial conditions. This decay is the physically expected behaviour in the absence of any forcing terms, but as may easily be shown for larger initial $(b, v)$, there is blow up at finite time $t=t_{0}$, asymptotically:

$$
v \sim\left(t_{0}-t\right)^{-1}, \quad b \sqrt{\ln b} \sim\left(t_{0}-t\right)^{-1}
$$

Analysis gives the stability criterion

$$
R m_{2}^{2}+\frac{1}{p_{m}} S m_{2}^{2}<\mathcal{O}(1)
$$

where the dimensionless parameter known as the mesh Reynolds number $R m_{s}=H\|u\|_{s} / \nu$, is calculated in terms of the $s$-norm of the initial $u$ and similarly the mesh Lundquist number $S m_{s}=H\left\|u_{\text {Alfven }}\right\|_{s} / \eta$.

Since the obvious objection to all the above original analysis is that it is based on models which are low order and/or heuristic, further investigations were pursued. 


\section{Nonlinear instability in Burgers' Equation}

The approach adopted was to solve a simpler problem than RMHD, but to do so rigorously and consider large $N$ th order systems, corresponding to discretisations on $N$ grid-points. The simplest nonlinear model for fluid dynamics is Burgers' equation for advection and diffusion of the velocity field $u(x, t)$ :

$$
u_{t}+u u_{x}=u_{x x}
$$

It is possible to solve Eq. (3.1) analytically by means of the Cole-Hopf transformation, which shows that all spatially periodic solutions must decay to $u=$ const., but also gives the weak, steady solution $u=\tan x$.

Burgers' equation may be written in semi-discrete form as

$$
u_{j}=N_{j}\left(u_{0}, \ldots, u_{N-1}\right)+L_{j}\left(u_{0}, \ldots, u_{N-1}\right), \quad j=0, . ., N-1,
$$

where specifically

$$
N_{j}=-u_{j}\left(u_{j+1}-u_{j-1}\right), \quad L_{j}=u_{j+1}-2 u_{j}+u_{j-1}
$$

The relevant dimensionless parameter is again $R m_{s}$, where $\|u\|_{s}$, is evaluated using the initial velocity values $u_{j}$ on the spatial grid.

Investigations by Eastwood \& Arter (1987b) and Davidson (1995) showed that the above choice of $N_{j}$ and $L_{j}$ led to generic behaviour, unless the nonlinearity $N_{j}$ was such as to conserve energy in the absence of dissipation, i.e. if it arose either from a specially constructed f.d. method, or from a spectral method. As anticipated in Section 1, the exact form of the boundary conditions was also found to be unimportant, and all results produced are for periodic boundaries $u_{0}=u_{N}$ unless stated otherwise.

It was found that, as in the low order models, both decaying solutions and solutions diverging as $\left(t_{0}-t\right)^{-1}$ were possible depending on initial conditions. In addition, the boundary in phase-space between the two types was found to be associated with the presence of fixed points of the $N$ th order system Eq. (3.2).

One of Davidson's major contributions was to give analytic expressions for these fixed points in $N$ dimensions. For the case of periodic boundaries with a zero mean flow:

$$
\begin{aligned}
u_{j} & =\tan \left(\frac{q \pi}{N}\right) \tan \left(\frac{q \pi}{N} \bar{j}\right) \\
\bar{j} & =j+\frac{1}{2} \quad(N \text { even }), \text { else } \bar{j}=j
\end{aligned}
$$

for integer $q$. This ties up with the previously obtained numerical fixed point solution as shown in Figure 2. Eq. (3.4) also generalises to the case when there is a mean flow, suppose this is of amplitude $\tan \left(\frac{\pi}{N}\right) \tan \left(\frac{\epsilon \pi}{2}\right)$ for some real $\epsilon$, then Davidson gives

$$
u_{j}=\tan \left(\frac{\pi}{N}\right) \tan \left(\frac{\pi}{N} \bar{j}+\frac{\epsilon \pi}{2 N}\right)
$$

The following solutions by Davidson reflect the fact that qualitatively similar phase space behaviour is found for the case of zero gradient boundary conditions, viz.

$$
\begin{aligned}
& u_{j}=-\tanh \left(\frac{A}{N}\right) \tanh \left(\frac{A}{2 N} j+\phi\right) \\
& u_{j}=-\tanh \left(\frac{A}{N}\right) \operatorname{coth}\left(\frac{A}{N} \bar{j}+\phi\right)
\end{aligned}
$$

Of note is the fact that whilst they lie close to the edge of the stability boundary, the fixed points do not determine it precisely. There are solutions with as much as $12 \%$ 


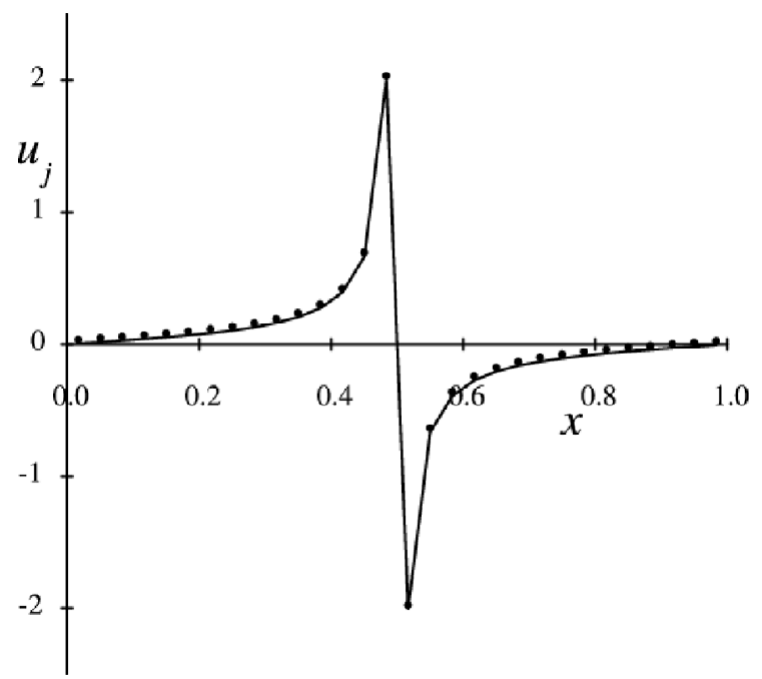

Figure 2. $u_{j}$ plotted against $x=\left(j+\frac{1}{2}\right) / N$ for the fixed point of the discrete Burgers' equation with $N=30$. The solid line joins points of the analytic expression Eq. (3.4), $q=1$.

smaller 2-norm or energy (although no smaller) than that possessed by the points Eq. (3.4) which do diverge. In addition, since the fixed points have at least one large jump, it is important to specify which norm is used to measure the initial velocity field. For example, as $N \rightarrow \infty$, the unstable fixed point approaches zero in the energy norm as $R m_{2} \rightarrow 2 \pi / \sqrt{N}$, whereas it remains finite in the maximum norm $R m_{\infty} \rightarrow 2$.

\section{Nonlinear instability in Fluid Dynamics}

Extrapolating results from the simple, compressible one-dimensional system represented by Burgers' equation to higher dimensional, incompressible flows is questionable. Hence, Davidson (1995) analysed discretisations of the viscous Euler equations for twodimensional fluid flow. The main thrust of the work related to the 2-D f.d. scheme expressible as

$$
\begin{aligned}
\frac{d \omega_{j, k}}{d t} & +\left(\omega_{j+1, k}-\omega_{j-1, k}\right)\left(\psi_{j, k+1}-\psi_{j, k-1}\right) \\
& -\left(\psi_{j+1, k}-\psi_{j-1, k}\right)\left(\omega_{j, k+1}-\omega_{j, k-1}\right) \\
& =\omega_{j+1, k}+\omega_{j-1, k}+\omega_{j, k+1}+\omega_{j, k-1}-4 \omega_{j, k}
\end{aligned}
$$

and

$$
\psi_{j+1, k}+\psi_{j-1, k}+\psi_{j, k+1}+\psi_{j, k-1}-4 \psi_{j, k}=-\omega_{j, k}
$$

where $\omega$ is the vorticity and $\psi$ the velocity stream function.

Problems were studied in the cases where either zero or periodic boundary conditions were imposed in each co-ordinate direction, for mesh sizes up to $18 \times 18$, i.e. 18 points in each direction. It was also established that similar results applied for other f.d., pseudospectral (p.s.), mixed f.d./p.s and mixed f.d./spectral schemes for meshes up $15 \times 15$. The form of the phase space was typically found to resemble that for Burgers' equation, with an attracting region enclosing the origin. One result for arbitrary $N$ was established, namely that solutions were proven to converge (i.e. decay) if the initial discrete velocity 
(i)

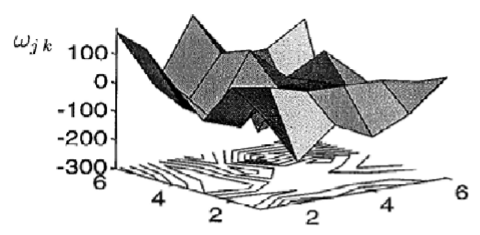

(iii) (ii)

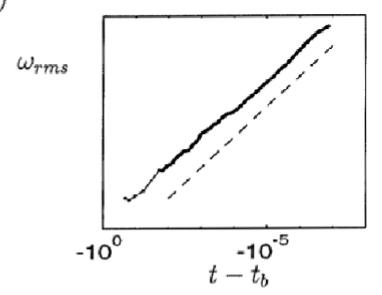

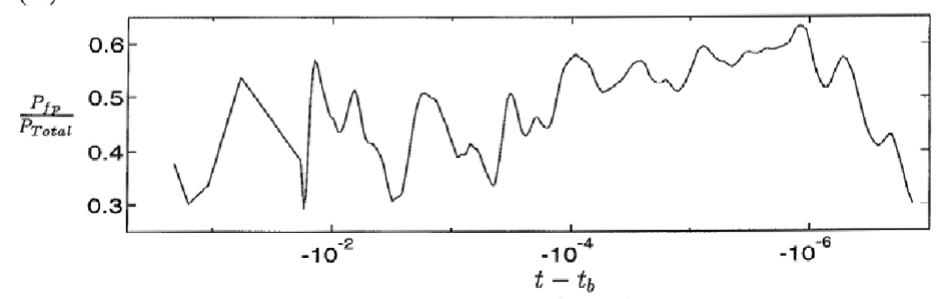

Figure 3. $6 \times 6$ mesh investigation, showing (i) initial conditions, (ii) blow-up in vorticity measure on log-log plot, and (iii) the power in the fixed point direction relative to the total power as the calculation diverges.

components satisfied

$$
\left|u_{j, k}\right| \leqslant 1 \text { and }\left|v_{j, k}\right| \leqslant 1
$$

corresponding to a restriction on $R m_{\infty}$.

Although a general formula for fixed points could not be found, it was possible to deduce exact expressions from some of the numerically calculated points, e.g. in the case of a $6 \times 6$ mesh:

$$
\omega_{j, k}(f p)=\left(\begin{array}{cccccc}
30 & 0 & 0 & 0 & 0 & 0 \\
30 & 0 & 0 & 0 & 0 & 0 \\
0 & -30 & -30 & 0 & 0 & 0 \\
0 & 0 & 0 & 30 & 0 & 0 \\
0 & 0 & 0 & 30 & 0 & 0 \\
0 & 0 & 0 & 0 & -30 & -30
\end{array}\right)
$$

A measure of the complexity of the work is the fact that the $6 \times 6$ system of equations, which is the smallest model exhibiting behaviour typical of larger systems, is of 34 th order. Figure 3 shows an indicative numerical calculation in this case. with a solution diverging as $\left(t_{0}-t\right)^{-1}$. The principal difference between the vorticity equation and Burgers' equation seems to be that, whereas the singular solutions to Burgers' are stable, those for the vorticity equation admit unstable directions (Davidson, 1995). This is supported by the fact that in Figure 3(iii) not all the power finishes in the direction of the fixed point. As in the case of Burgers' equation, the most dangerous fixed points have large jumps. Davidson has estimated that the fixed point satisfies $R m_{2} \approx 59.7 / \sqrt{N}$ as $N \rightarrow \infty$ whereas $R m_{\infty} \rightarrow 41.7$. Criteria of the same form with similar magnitude estimates were also found for the other schemes mentioned above. In respect of $R m_{\infty}$ criteria, the stability of the vorticity equation is better than Burgers', but it remains uncertain whether this also holds true for RMHD. These stability results should be compared with the criterion for the accuracy of fluid calculations derived by Kreiss, see the review by Arter (1995). Kreiss's criterion may be put into the form $R m_{\infty} \leqslant \pi^{2}$ (Davidson, 1995). 


\section{Discussion}

Alias-feedback instabilities are not believed to be a problem for most present-day codes, which are normally designed so that the total energy cannot increase due to nonlinear interactions. In any event, satisfying accuracy criteria which limit the mesh Reynolds' and Lundquist numbers to order unity, should prevent the nonlinear numerical instability.

Nonetheless, the work described herein, particularly that due to J. Davidson, is still of value in that it indicates how difficult it is likely to be to establish by purely numerical means, the presence of finite time singular behaviour in fluid dynamics, see e.g. Hou (2009), Moffatt (2009) for background. It has also been shown that results obtained for more general flows are qualitatively similar to ones obtained for Burgers' equation. Now, applied to Burgers' equation, the energy conserving schemes like spectral methods are incapable of representing the steady singular solutions. As such a solution does not exist, it cannot be attracting and so a finite time singularity in the limit of vanishing timestep cannot be found by these methods.

Therefore the suggested way forward is to seek additional singular solutions in 2-D and 3-D of schemes for fluid dynamics which are not semi-conservative. These discrete solutions might be indicative, like the discrete tan and coth solutions found for Burgers' equation, of the functional form of weak solutions to Navier-Stokes'. It may then be possible to understand if or how such weak solutions can be produced, and whether they are unstable like the 2-D singular solutions found by Davidson (1995).

\section{Acknowledgements}

This work was funded by the United Kingdom Engineering and Physical Sciences Research Council under grant EP/G003955 and the European Communities under the contract of Association between EURATOM and CCFE. The views and opinions expressed herein do not necessarily reflect those of the European Commission.

\section{References}

Arter, W., Plasma Physics and Controlled Fusion, To be submitted, 2010.

Arter, W., Reports on Progress in Physics, 58:1-59, 1995.

Arter, W. \& Eastwood, J. W., Transport Theory and Statistical Physics, 16:433-446, 1987.

Davidson, J., Dynamics of semi-discretised fluid flow, 1995. Dissertation submitted to the University of Cambridge.

Eastwood, J. W. \& Arter, W., Physical Review Letters, 57:2528-2531, 1986.

Eastwood, J. W. \& Arter, W., Physics of Fluids, 30:2774-2783, 1987a.

Eastwood, J. W. \& Arter, W., I.M.A. Journal of Numerical Analysis, 7:205-222, 1987b.

Hazeltine, R. D. \& Meiss, J. D., Plasma Confinement. Addison-Wesley, Redwood City, 1992.

Hou, T. Y., Acta Numerica, 18:277-346, 2009.

Moffatt, H. K., In R.L. Ricca, editor, Lectures on Topological Fluid Mechanics, pages 157-166. Springer Verlag, 2009.

Proctor, M. R. E., In A.M. Soward, C.A. Jones, D.W. Hughes, and N.O. Weiss, editors, Fluid dynamics and dynamos in astrophysics and geophysics, pages 235-278. CRC, 2005.

Tritton, D. J., Physical Fluid Dynamics, 2nd Edition. Clarendon Press, Oxford, 1988. 